\title{
Evaluating the Impact of Simulated Patients on Knowledge, Skills and Attitudes of Laypeople Following a Basic First Aid Course: Protocol for a Cluster-Randomized Controlled Trial
}

Bert Avau, Anne-Catherine Vanhove, Marianne Vandenlindenloof, Liselotte Suls, Hans Verstraeten, Philippe Vandekerckhove, Emmy De Buck

Background: Teaching first aid to laypeople is a cost-effective way to improve public health. However, it is currently unclear what the most effective ways are to teaching first aid. It has already been shown that simulated patients have an added value in teaching emergency care to healthcare professionals. This paper describes the protocol for a cluster-randomized controlled trial that will investigate the influence of using a simulated patient during basic first aid training for laypeople on their knowledge, skills and self-efficacy.

Hypothesis: The null hypothesis of this research is that the use of simulated patients during basic first aid training does not result in a statistically significant change in knowledge, skills and self-efficacy concerning the first aid topics for which a simulated patient is used. The alternative is that knowledge, skills and self-efficacy will be influenced by using simulated patients during training.

Methods: This study will be a cluster-randomized controlled trial that will take place from September 2018 to June 2020. The study population will consist of employees from several organizations taking a three-day first aid certification course with the Belgian Red Cross. The organizations requesting a first aid course will be randomized in receiving a course with or without simulated patient. The simulated patient will only be used for the topics "first aid for burns" and "first aid for stroke" in the intervention group. The participants will complete a questionnaire to measure their knowledge and self-efficacy and complete a practical skills test after the course testing these first aid topics. To test whether the retention of first aid knowledge and self-efficacy is influenced by using a simulated patient during a certification course, participants following a refresher course one year after the certification course will complete a follow-up questionnaire.

Conclusion: This cluster-randomized controlled trial will be, to our knowledge, the first to investigate the added value of simulated patients during first aid courses for laypeople.

\section{Background}

Teaching first aid to laypeople is, according to the World Bank, a very cost-effective way to improve public health in low and middle income countries (Laxminarayan et al., 2006). The World Health Organization (WHO) estimates that the global number of deaths due to injury was close to 5 million in 2015 (WHO, 2016). Not surprisingly, large efforts are being made to improve the layperson's capacity to provide first aid and thereby increase a population's resilience (He, Wynn, \& Kendrick, 2014; Kleinman et al., 2018; Van de Velde et al., 2009). This is also true in developing regions, where access to professional healthcare is limited, and prehospital care by first responders may be even more important than in 
developed countries (Geduld \& Wallis, 2011; Husum, Gilbert, \& Wisborg, 2003; Merchant et al., 2015).

Tannvik et al. conducted a systematic review to investigate how often laypeople provide first aid when confronted with an injury, the quality of the applied first aid interventions, and the impact of the first aid provided on the outcomes of the injury (e.g. mortality) (Tannvik, Bakke, \& Wisborg, 2012). A large heterogeneity between studies was found, with the frequency of first aid provided ranging from as little as $11 \%$ of cases to as much as $65 \%$. The quality of first aid provided was also variable: for some interventions up to $84 \%$ of the help that bystanders provided might have been inappropriate. Nevertheless, a retrospective cohort study suggested that mortality could decrease by $6 \%$ in trauma victims as a result of first aid application (Murad \& Husum, 2010). Furthermore, in a cohort of out-of-hospital cardiac arrest survivors, bystander cardiopulmonary resuscitation (CPR) was associated with improved cerebral function (Abe, Tokuda, \& Ishimatsu, 2009).

Despite these results, relatively little is known about the most effective methods to teach first aid to laypeople. A systematic review by $\mathrm{He}$ et al. demonstrated a paucity in high-quality trials that demonstrate the effectiveness of first aid education for laypeople (He et al., 2014). On the other hand, a more realistic experience, via some form of simulation training, can have an added value in the training of healthcare professionals providing prehospital care (Abelsson, Rystedt, Suserud, \& Lindwall, 2014, 2016).

High-fidelity simulation training can be provided by using manikins or simulated patients (real-life actors). Several studies have already compared the use of manikins to simulated patients for training healthcare professionals in emergency care, including trauma teaching (Ali, Al Ahmadi, Williams, \& Cherry, 2009; Lee et al., 2003; Wisborg, Brattebo, BrinchmannHansen, \& Hansen, 2009), acute care scenarios (Coffey et al., 2016) and advanced cardiac life support (Miotto, Couto, Goulart, Amaral, \& Moreira Mda, 2008). In the study by Lee et al., surgery interns had better trauma assessment scores when training with manikins, compared to simulated patients (Lee et al., 2003). In contrast, Ali et al. concluded that skills and knowledge in trauma evaluation of final year medical students improved equally when using manikins or simulated patients (Ali et al., 2009), while Wisborg et al. found that hospital trauma teams valued the educational level of training with manikins and simulated patients equally, but had a slight preference for simulated patients in scenarios where interaction with the patient was necessary (Wisborg et al., 2009). Coffey et al. investigated the verbal and non-verbal interactions of clinical teams with manikins and simulated patients in acute care scenarios and concluded that the number of interactions was much larger when dealing with simulated patients (Coffey et al., 2016). Whether this also influenced the actual clinical performance of the team was not investigated in this pilot study. Finally, Miotto et al. could not demonstrate the added value of using simulated patients in advanced cardiac life support courses (Miotto et al., 2008). Of course, it needs to be taken into account that for more invasive procedures used in advanced cardiac life support, it is not possible to use real-life patients.

In addition to these studies, Herbstreit et al. compared the use of simulated patients to traditional seminars during training of medical students in the management of different acute care scenarios (Herbstreit et al., 2017). An educational approach with simulated patients resulted in improved skills, but similar knowledge to traditional training.

To our knowledge, no studies have been conducted so far to investigate the added value of simulated patients during first aid education for laypeople.

With this study, we aim to fill this knowledge gap, by comparing participants taking a first aid certification course, with or without the use of real-life simulated patients. At the moment no standard set of outcomes for educational interventions has been established by the COMET initiative (Gargon, 2016). The outcomes knowledge, skills, behavior and confidence are however widely used in education intervention studies and first aid education intervention studies specifically (He et al., 2014; Krathwohl, 2002; Simpson, 1966). Studying actual helping behavior using deception trials is beyond the scope of this study. We will measure self-efficacy 
specifically, rather than the more global self-belief or confidence. Self-efficacy was defined by Bandura as a person's beliefs in their own capabilities to perform specific actions and is responsive to instructional interventions (Bandura, 1977, 2006a; Zimmerman, 2000). Efficacy beliefs influence amongst others people's actions, their outcome expectations of said actions and whether they persevere when they face obstacles (Bandura, 1977, 2006b). In our study, the use of simulated patients in specific topics in the first-aid course could give the participants a learning experience more closely resembling an actual emergency which could enhance their self-efficacy for those types of first aid interventions. We will therefore compare the knowledge, skills and self-efficacy concerning the first aid topics burns and stroke, of participants taking a first aid certification course, with or without the use of reallife simulated patients for the topics burns and stroke.

\section{Methods}

\section{Research question}

This study aims to evaluate whether using a simulated patient for the topics burns and stroke, compared to not using a simulated patient, during an occupational first aid certification course influences knowledge, skills and self-efficacy of the participants concerning first aid for burns and stroke. The comparison, a course without simulated patients, is how the majority of our (Belgian Red Cross) courses are taught, making this an appropriate comparator.

The null hypothesis is that there will be no statistically significant difference in participant's knowledge, skills and self-efficacy after a first aid certification course with a simulated patient, compared to a course without, both immediately after the course and after 1-year follow-up. The alternative hypothesis is that there will be a statistically significant change in knowledge, skills and self-efficacy between participants following a first aid certification course with or without a simulated patient, both immediately after the course and/or after 1-year follow-up.

\section{Participants}

In Belgium, the presence of employees skilled in first aid within an organization is regulated by law, depending on the type of economic activity and the size of the organization (FOD Werkgelegenheid Arbeid en Sociaal Overleg, 2011). For example, organizations with an assumed average risk of injury (e.g. assembly companies) employing between 20 and 50 people are required to have one person trained in first aid present at all times. Workplace first aiders are initially trained by a recognized first aid training provider in a first aid certification course (18 hours training +3 -hour exam) and follow a yearly 4-hour refresher course. The obligation to follow a yearly refresher course enables a longitudinal investigation of the effectiveness of the provided first aid courses. The Belgian Red Cross is a major provider of both the initial certification and refresher first aid courses, therefore having the opportunity to standardize the content and format of the courses provided, making participants of these courses a convenient and appropriate population to study. As this is a convenience sample, we will take prior first aid training, age, gender, level of education and prior experience with first aid situations into account as potential confounding factors during the statistical analyses.

\section{Study design}

\section{Ethical considerations}

This study has been evaluated and approved of by the Social and Societal Ethical Committee (SMEC) of Leuven University (KU Leuven), Belgium, with following file number: G- 201806 1273. We have registered the trial at Clinicaltrials.gov as NCT03608982. We reported this protocol paper according to the guidelines of the SPIRIT (Standard Protocol Items: Recommendations for Interventional Trials) statement (Chan et al., 2013). A completed SPIRIT checklist can be found in appendix 1.

Participants will complete an informed consent form for agreement and participation in a study to evaluate the first aid courses taught by the Belgian Red Cross (see appendix 2). Any data that we will collect from trial participants will be considered confidential. We will anonymize questionnaires upon entry in our database, except for a unique participant and organization number. We will analyze and report the data anonymously. We will destroy the key linking 
participant identity and outcome data after data from the initial assessment and data from assessment after one year are linked together for each individual.

\section{Randomization $\&$ intervention allocation}

This is a cluster-randomized controlled superiority trial. The unit of randomization in this study is an organization requesting a first aid course, rather than the individual participant. As some organizations will request more than one course, this will be a clusterRCT with two levels of clustering, the organization and the course. We will assign organizations randomly in a 1:1 ratio to a first aid course with simulated patient (intervention) or without (control) via block randomization with variable block sizes (2 or 4 ). A researcher who is not involved in the practical organization of the first aid courses (BA) will generate the randomization list via an online tool (http://www.randomization.com). The randomization scheme will be contained in sequentially numbered, sealed, opaque envelopes and therefore kept concealed up to the moment an organization is allocated to the intervention or control group by administrative employees of the Belgian Red Cross, occupied with the practical organization of the courses.

\section{Blinding}

Blinding of interventionists, i.e. first aid instructors and simulated patients, is not possible, due to the nature of the intervention. We will keep the participants and their employers blinded to the aims of the study but will provide an informed consent and inform them that their scores during examination will be used to evaluate the performance of the Belgian Red Cross first aid trainings. We will use a strict protocol for the courses, thus limiting the potential influence of lack of blinding of the interventionists. The outcome skills will be assessed by researchers not involved in the organization of the courses, who are kept blinded to the allocation status of the participants they score. We will enter scores in a database and will reveal the allocation status for analysis purposes after all final scores are inserted in a data collection database.

\section{Interventions studied}

\section{Setting}

The first aid certification courses of the Belgian Red Cross are $18 \mathrm{~h}(+3 \mathrm{~h}$ exam) courses on a range of first aid topics, e.g. cardiopulmonary resuscitation, burns, wounds, etc. The courses are taught during three working days spread over three weeks. On average, there are 10 participants per basic first aid course. The people following a first aid certification course in Dutch in the period September 2018 to June 2019, ordered by their employer at the Belgian Red Cross will be eligible for this trial. For the purpose of this study, organizations ordering a first aid course will not be offered the choice of a course with or without a simulated patient but will be randomized in receiving a course with or without a simulated patient, free of charge. Organizations that purposefully request a simulated patient will not be eligible for participation in this trial, as this would break randomization. Organizations requesting a course in a language other than Dutch (e.g. French, English) will not be eligible for inclusion.

Participants from organizations that order a refresher course in the year following the first aid certification course will be eligible to measure one-year retention of feasibility issues. As we aim to measure retention after one year, organizations that book a refresher course sooner than 10 months or later than 14 months after the certification course will not be eligible for retention measurements. See figure 1 for a schematic overview of the trial.

\section{Intervention: first aid certification course with simulated patient}

The materials in this course are standardized, and consist of lectures with a slide show, questions \& answer conversations, and practical exercises on fellow students. The topics that are being taught during the courses are the following: principles of first aid, 
Figure 1: Overview of Proposed Trial

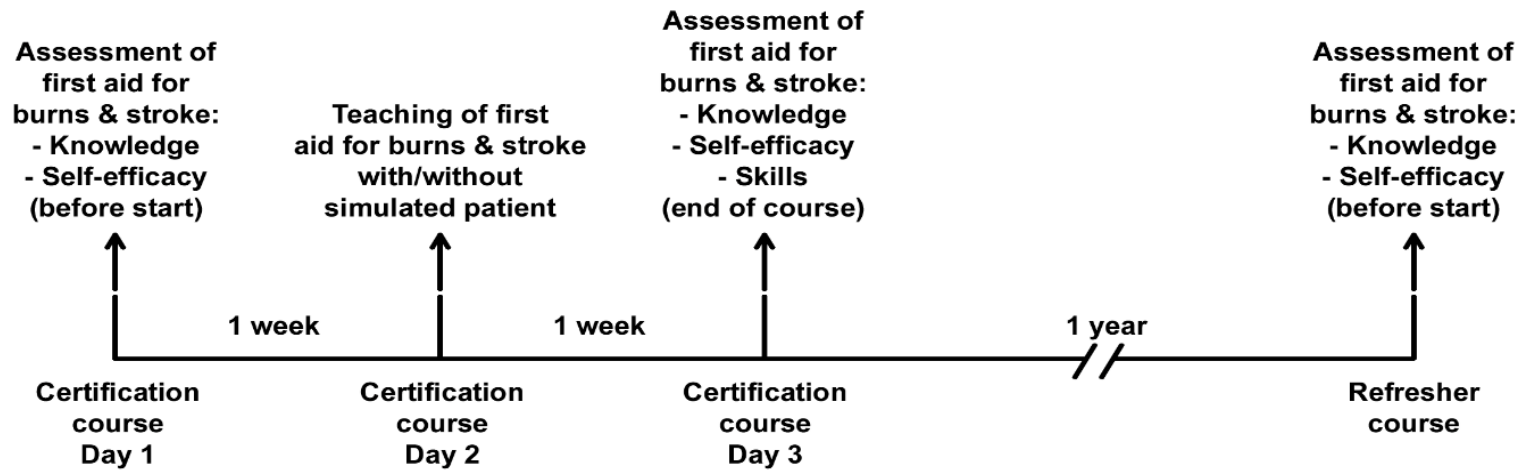

cardiopulmonary resuscitation, first aid for bleeding and skin wounds (day 1), first aid for burns, syncope, stroke, head and neck injuries, eye injury, epilepsy, chest pain, respiratory problems, choking and poisoning (day 2), first aid for injuries to bones, muscles and joints, blisters, diabetes, the legal framework for first aid and a reiteration (day 3). The courses are being taught by professional first aid instructors from the Belgian Red Cross. During the practical exercises, after participants have received the theoretical explanations by the instructor, a simulated patient will unexpectedly enter the room requiring treatment. This simulated patient is a professional actor and Belgian Red Cross employee, who combines acting distress and pain with make-up, to mimic the injuries as truthfully as possible. The instructor will ask two participants to pretend this is an actual first aid emergency and to demonstrate how they would care for the simulated patient, according to what has been taught during the theoretical part of the course. The simulated patient will respond as described below. However, the instructor will closely follow the caring by the two participants, to guarantee the safety of the simulated patient. The instructor is also the one who determines when the simulation exercise ends. After the exercise, a group discussion follows on how the two volunteers diagnosed and/or managed the simulated patient, what they did well and what could have been better. In this discussion, both the volunteers and the simulated patient, as well as the other participants will be questioned by the instructor on how they experienced the exercise.

For feasibility reasons, we will include the simulant in only two sections of the course: the respective treatments of burns and stroke. These first aid topics

will take place on the second day of the three-day course. The remainder of the course will be taught without simulated patient by the professional first aid instructors. The simulated patient will follow a standardized routine during his intervention, as follows:

- For the burns topic, the simulated patient will enter the room with first and second degree burns on the inside of the lower left arm. The area which is burned to the first degree will show erythema (redness, using make-up), and will be approximately $14 \mathrm{~cm}$ long and $4 \mathrm{~cm}$ wide. Inside this area, there will be three to four second degree burn blisters of approximately $1 \mathrm{~cm}^{2}$ (made with make-up). The simulated patient will feign pain in the burned area, which worsens when being touched during cooling and covering of the burn.

- For the stroke topic, the simulated patient will be present in the room at the start of this part of the curriculum and will demonstrate a hemiparalysis of the right part of the body. To do so, the simulated patient will be sitting on a chair, showing weakness in the right side of the body and supporting herself by leaning on the table with the left part of the body. The left foot will be placed firmly on the ground. The right arm will be dangling next to the body. The right foot will be tilted. The head will tilt to the right side and the right tip of the mouth will show central facial palsy. The simulated patient will use the left hand to support the left side of the head. When asked how she feels, the simulated patient will demonstrate speech difficulties and complain of 
a headache. The FAST-test (Face-Arm-SpeechTime), which is recommended for initial diagnosis of stroke (Zideman et al., 2015), will be positive when performed by the participant on the simulated patient: facial droop will remain present when asked to smile or show teeth, right arm will drift or not be lifted when asked to raise both arms and speech will remain slurred.

Burns and stroke were the topics of choice for this trial, as we consider them to be complementary to each other: Burns are mostly non-life-threatening, but require a fair number of practical actions to be treated in a first aid setting (i.e. cooling in an appropriate fashion, application of a hydrating crème, application of a bandage), while stroke is a life-threatening disorder, but requires fewer practical actions to be treated in a first aid setting (i.e. only performing the FAST-test and calling the emergency medical services).

\section{Control: first aid certification course without simulated patient}

The course materials in the control courses are also standardized, with identical content as the course materials in the intervention courses. Instead of using simulated patients, however, we will use video clips and case photographs to demonstrate the first aid techniques.

\section{Outcomes of interest}

To define primary outcomes for this trial, we have searched the website of the COMET initiative, which tries to define a minimum set of core outcomes to be reported in trials for a specific disorder (Gargon, 2016). A set of core outcomes does not seem to exist for educational interventions, however skills, knowledge and self-efficacy are widely used as key determinants of effective learning (Krathwohl, 2002; Simpson, 1966; Zimmerman, 2000). Therefore, these outcomes will be the primary outcomes of this review.

Before the course, we will assess participants' first aid knowledge and self-efficacy in providing first aid. In addition, we will question following demographic parameters: age, gender, educational level, whether a previous first aid course was followed and whether the person had a previous experience of first aid.
After completing the course, we will test knowledge, skills and self-efficacy. In addition, we will verify retention of knowledge and self-efficacy after one year by testing participants who follow another yearly refresher course in the coming year. We will not measure the outcome skills before the course and after one year, due to feasibility issues.

Secondary outcomes of this course will be participant satisfaction with the course received and costs associated with the use of simulated patients.

We will test participants for knowledge, skills and selfefficacy as part of their examination, at the end of the three-day course. The participants will complete the self-efficacy and subsequent knowledge assessment questionnaires prior to the practical skills test.

We will measure knowledge using a short multiplechoice questionnaire of 10 questions, testing different concepts taught during the two topics where a simulant was present or absent, which can be answered either correctly or falsely. In addition, we will blend in 10 questions on first aid topics taught during the certification course but not related to the use of a simulated patient to keep participants blinded to the actual goal of the research. The questionnaire used can be found in appendix 3 .

We will measure self-efficacy also using a short questionnaire (appendix 4). The aim of this questionnaire is to evaluate how confident a participant is in his or her capacities to provide first aid when necessary. The questionnaire consists of six questions on the participant's self-efficacy to provide first aid in case of burns or stroke, answered on a 5-point Likert scale (O'Muircheartaigh, A. Krosnick, \& Helic, 2000), and we have based it on a previously designed and validated questionnaire (Wei et al., 2013). To keep participants blinded, we will blend these questions in with six questions concerning their self-efficacy to provide first aid for other topics taught during the course.

We will measure skills by recording a practical test which will be scored via a checklist with 14 skills (eight for burns and six for stroke). Participants can achieve a total maximal score of 18 (nine for burns and nine for 
stroke), where the score available for each individual skill depends on the perceived criticalness of the skill assessed, as judged by the Belgian Red Cross's First Aid Service. For example, when treating burns, we consider the skill 'cooling with water' critical, for which it counts for 5 out of 9 points to be earned. The skill 'applying a hydrating crème' is considered less critical, for which it counts for only 1 out of 9 points to be earned. The checklists and scores to be earned for the individual skills are shown in appendix 5 . In short, for first aid for burns, we will judge participants on hygiene measures taken, appropriate judgment of the need for specialized help, appropriate cooling of the burn, application of a hydrating crème and appropriate covering of the burn. For first aid for stroke, we will judge participants on providing safety for the victim, adequate performance of the FAST test and immediate alerting of the emergency services. The participants will apply skills to the first aid instructor, which will be recorded with a camera and judged afterwards independently by two assessors who are not involved in the organization or conduct of the courses that are being assessed. These assessors will not be aware of the allocation status of the participants they judge. Outcome assessors will report if for some reason blinding of the allocation status of a participant is broken. If a discrepancy exists between the two assessors, we will involve a third researcher. Participants for first aid certification courses are usually tested on skills concerning cardiopulmonary resuscitation (legal obligation), two life-threatening conditions and two non-life-threatening conditions. For the purpose of this study, one life-threatening condition and one non-life-threatening condition tested will be stroke and burns, respectively. The first aid instructors will present fixed cases of burns and stroke, to make sure the exams are as standardized as possible. The burn case to be treated will be a first degree burn on the lower left arm. The participants will have access to the standard content of a first aid kit (e.g. gloves, bandages, plasters, hydrating crèmes, disinfectants etc.), in addition to water and a receptacle, which can be used to irrigate the burn with water. The stroke case will be demonstrated in a similar fashion as described for the simulated patient. To blind the participants for the actual goal of the research, we will ask them to pick cards from a stack to determine which practical skills they need to demonstrate.

We will assess the secondary outcome, participant satisfaction, by asking the participants to score their satisfaction with the course provided (without specifying the use or non-use of a simulated patient) on a 10-point scale. We will record the other secondary outcome, costs involved, during the study and express this relatively to the gain in the primary outcomes-knowledge, skills and self-efficacy.

We have tested the validity and comprehensibility of our questionnaire on first aid self-efficacy during first aid certification courses taught in the spring of 2018. The questionnaire was completed before and after following a first aid certification course by a total of 79 participants, divided over 7 courses. Participants found the questions clear, and the internal validity of the selfefficacy questionnaire was demonstrated by a Cronbach $\alpha$ of 0.87 .

\section{Data collection and management procedures}

The first aid instructions will collect the demographic data and knowledge and self-efficacy outcomes using questionnaires. We will collect results from the skills assessment via checklists. A researcher will anonymize and insert the data in a database (LS and MV). Researchers not involved in the practical organization or conduct of the courses (BA and AV) will perform analyses on anonymized data. These researchers will also perform range checks on the data provided.

Researchers who are not involved in the practical organization or conduct of the courses (BA and AV) will perform periodical (monthly) monitoring of the study's conduct.

\section{Statistical methods}

\section{Sample size calculation}

We have calculated a required sample size to obtain $90 \%$ power at the 0.05 significance level, taking into account the clustered design of our study. We have used following formula, proposed by Teerenstra et al. (Teerenstra, Moerbeek, van Achterberg, Pelzer, \& Borm, 2008), but re-expressed according to Rutterford et al. (Rutterford, Copas, \& Eldridge, 2015): 


$$
m=\frac{\left(Z_{1-\frac{\alpha}{2}}+Z_{1-\beta}\right)^{2} 2 \sigma^{2}}{\Delta^{2}}\left(1+n_{1}\left(n_{2}-1\right) \rho_{2}\right.
$$

Where:

$\mathrm{m}=$ the number of participants required in each trial arm

$\mathrm{Z}_{1-\alpha / 2}=\mathrm{Z}$-value associated with the chosen confidence level (0.05)

$\mathrm{Z}_{1-\beta}=\mathrm{Z}$-value associated with the chosen power level $(0.9)$

$\sigma^{2}=$ total variance in the primary outcome

$\Delta=$ anticipated difference between intervention and control group

$\mathrm{n}_{1}=$ average amount of participants per course

$\varrho_{1}=$ intracluster correlation coefficient for participants

in a course

$\mathrm{n}_{2}=$ average amount of courses per organization

$\varrho_{2}=$ intracluster correlation coefficient for courses in an organization

We have defined the primary outcome of interest to be the difference in one-year retention of self-efficacy concerning first aid between participants following a first aid certification course with or without a simulated patient. We have estimated the total variance in this outcome $\left(\sigma^{2}=0.75\right)$ and intracluster correlation coefficient $\left(\varrho_{1}=0.025\right)$, based on the results obtained during the pilot phase of our self-efficacy questionnaire, in the spring of 2018. As the first aid certification courses in which we piloted the questionnaires were organized in distinct organizations, no estimate is available for $\varrho_{2}$, for which we assume this to be similar to @1, i.e. 0.025. A sample size calculation based on these previous courses and data from the pilot phase suggests an initial required sample size of 496 potential participants to detect a $5 \%$ increase in selfefficacy with $90 \%$ power at the 0.05 significance level.

We anticipate a drop-out of $41 \%$ percent after one year, due to organizations not ordering refresher courses or organizations ordering refresher courses less than 10 months or more than 14 months after ordering the certification course and another $30 \%$ at participant level due to employees refusing to participate or not following the refresher course after one year. These numbers are based on data we collected from a sample of 482 organizations that followed Belgian Red Cross first aid certification and refresher courses over the past 3 years. We will try to limit the loss to follow-up after one year by actively inviting organizations to organize refresher courses promptly. On average, 10 participants participated per course, and 1.35 courses were organized per organization over the past three years. Taking into account initial refusal and drop-out, the required sample size is 1202 , which is an attainable number and goal during the anticipated recruitment period.

\section{Statistical tests to be used}

We will perform a per-protocol analysis. To test a difference in knowledge, skills or self-efficacy, immediately after the course, and a difference in knowledge and self-efficacy, 1 year after the course, we will build a (generalized) linear mixed model with intervention as main variable. We will take into account participant age, participant gender, participant educational level, whether the participant followed a previous first aid course and whether the participant had previous experience with first aid as fixed effect covariates. Course, organization and the interaction of course and organization will be added as random effects in the model. We will conduct statistical analyses using the open source software of the R-project for statistical computing, version 3.2.5 (R Core Team, 2016). We will only analyze complete cases. We will accept statistical significance at the $5 \%$ level. We will present data as means with standard deviations, and as summary effects using mean differences with 95\% confidence intervals.

\section{Dissemination plans}

We will disseminate the results of this study via publication in a peer-reviewed journal and by communication via social media. Participants, who provided their e-mail address on the participation form, will be emailed a report containing the results. We are as yet undecided whether we will provide full access to individual participant data. We will disseminate substantial amendments to the protocol to relevant parties, including the trial registry. Researchers who deliver a substantial intellectual contribution to the 
work will be eligible as co-author. We do not intend to make use of professional writers.

\section{Sources of support}

This work is funded in part by the Foundation for Scientific Research of the Belgian Red Cross (Motstraat 42, 2800 Mechelen, Belgium), by internal funds from the First Aid service of the Belgian Red Cross and by a grant from the King Baudouin Foundation.

The external sponsor had no role in study design, collection, management, analysis and interpretation of data; writing of this report and the decision to submit the report for publication, nor will this be the case during the future course of the trial.

\section{Declarations of interest}

All authors are employees of the Belgian Red Cross and have no further interests to declare.

\section{Author contributions}

BA: Designed the research, will coordinate the trial, will perform data analysis, will interpret the data, wrote the draft manuscript of the protocol paper, will write the draft manuscript of the results paper.

AV: Designed the research, will coordinate the trial, will perform data analysis, will interpret the data, wrote the draft manuscript of the protocol paper, will write the draft manuscript of the results paper.

MV: Will coordinate the trial, will collect data, will perform data analysis, will interpret the data, revised the manuscript of the protocol paper, will revise the manuscript of the results paper.

LS: Will coordinate the trial, will collect data, revised the manuscript of the protocol paper, will revise the manuscript of the results paper.

HV: Conceptualized the research, will supervise the trial, will interpret the data, revised the manuscript of the protocol paper, will revise the manuscript of the results paper.

EDB: Conceptualized the research, will supervise the trial, will interpret the data, revised the manuscript of the protocol paper, will revise the manuscript of the results paper.

PV: Conceptualized the research, will supervise the trial, will interpret the data, revised the manuscript of the protocol paper, will revise the manuscript of the results paper

\section{References}

Abe, T., Tokuda, Y., \& Ishimatsu, S. (2009). Predictors for good cerebral performance among adult survivors of out-of-hospital cardiac arrest. Resuscitation, 80(4), 431-436. doi:10.1016/j.resuscitation.2008.12.010

Abelsson, A., Rystedt, I., Suserud, B. O., \& Lindwall, L. (2014). Mapping the use of simulation in prehospital care - a literature review. Scand J Trauma Resusc Emerg Med, 22, 22. doi:10.1186/1757-7241-22-22

Abelsson, A., Rystedt, I., Suserud, B. O., \& Lindwall, L. (2016). Learning by simulation in prehospital emergency care - an integrative literature review. Scand J Caring Sci, 30(2), 234-240. doi:DOI: $10.1111 /$ scs.12252

Ali, J., Al Ahmadi, K., Williams, J. I., \& Cherry, R. A. (2009). The standardized live patient and mechanical patient models--their roles in trauma teaching. J Trauma, 66(1), 98-102. doi:10.1097/TA.0b013e318193ba84

Bandura, A. (1977). Self-efficacy: Toward a unifying theory of behavioral change. Psychological Review, 84(2), 191-215.

Bandura, A. (2006a). Guide for constructing self-efficacy scales. In F. Pajares \& T. Urdan (Eds.), Self-efficacy beliefs of adolescents (Vol. 5, pp. 307-337). Greenwich, CT: Information Age Publishing. 
Bandura, A. (2006b). Toward a Psychology of Human Agency. Perspectives on psychological science, 1(2), 164180.

Chan, A. W., Tetzlaff, J. M., Gotzsche, P. C., Altman, D. G., Mann, H., Berlin, J. A., . . Moher, D. (2013). SPIRIT 2013 explanation and elaboration: guidance for protocols of clinical trials. BMJ, 346, e7586. doi:10.1136/bmj.e7586

Coffey, F., Tsuchiya, K., Timmons, S., Baxendale, B., Adolphs, S., \& Atkins, S. (2016). Simulated patients versus manikins in acute-care scenarios. Clin Teach, 13(4), 257-261. doi:10.1111/tct.12425

FOD Werkgelegenheid Arbeid en Sociaal Overleg. (2011). EERSTE HULP OP HET WERK. Retrieved from http://www.werk.belgie.be/WorkArea/DownloadAsset.aspx?id=34480

Gargon, E. (2016). The COMET (Core Outcome Measures in Effectiveness Trials) Initiative. Maturitas, 91, 9192. doi:10.1016/j.maturitas.2016.06.007

Geduld, H., \& Wallis, L. (2011). Taxi driver training in Madagascar: the first step in developing a functioning prehospital emergency care system. Emerg Med J, 28(9), 794-796. doi:10.1136/emj.2010.101683

He, Z., Wynn, P., \& Kendrick, D. (2014). Non-resuscitative first-aid training for children and laypeople: a systematic review. Emerg Med J, 31(9), 763-768. doi:10.1136/emermed-2013-202389

Herbstreit, F., Merse, S., Schnell, R., Noack, M., Dirkmann, D., Besuch, A., \& Peters, J. (2017). Impact of standardized patients on the training of medical students to manage emergencies. Medicine (Baltimore), 96(5), e5933. doi:10.1097/MD.0000000000005933

Husum, H., Gilbert, M., \& Wisborg, T. (2003). Training pre-hospital trauma care in low-income countries: the 'Village University' experience. Med Teach, 25(2), 142-148. doi:10.1080/0142159031000092526

Kleinman, M. E., Goldberger, Z. D., Rea, T., Swor, R. A., Bobrow, B. J., Brennan, E. E., . . Travers, A. H. (2018). 2017 American Heart Association Focused Update on Adult Basic Life Support and Cardiopulmonary Resuscitation Quality: An Update to the American Heart Association Guidelines for Cardiopulmonary Resuscitation and Emergency Cardiovascular Care. Circulation, 137(1), e7-e13. doi:10.1161/CIR.0000000000000539

Krathwohl, D. R. (2002). A Revision of Bloom's Taxonomy: An Overview. THEORY INTO PRACTICE, 41(4), 212218.

Laxminarayan, R., Mills, A. J., Breman, J. G., Measham, A. R., Alleyne, G., Claeson, M., . . Jamison, D. T. (2006). Advancement of global health: key messages from the Disease Control Priorities Project. Lancet, 367(9517), 1193-1208. doi:10.1016/S0140-6736(06)68440-7

Lee, S. K., Pardo, M., Gaba, D., Sowb, Y., Dicker, R., Straus, E. M., . . Knudson, M. M. (2003). Trauma assessment training with a patient simulator: a prospective, randomized study. J Trauma, 55(4), 651657. doi:10.1097/01.TA.0000035092.83759.29

Merchant, A., Outhay, M., Gonzalez-Calvo, L., Moon, T. D., Sidat, M., Taibo, C. L., \& McQueen, K. (2015). Training laypersons and hospital personnel in basic resuscitation techniques: an approach to impact the global trauma burden in Mozambique. World J Surg, 39(6), 1433-1437. doi:10.1007/s00268-0152966-z 
Miotto, H. C., Couto, B. R., Goulart, E. M., Amaral, C. F., \& Moreira Mda, C. (2008). Advanced Cardiac Life Support Courses: live actors do not improve training results compared with conventional manikins. Resuscitation, 76(2), 244-248. doi:10.1016/j.resuscitation.2007.07.031

Murad, M. K., \& Husum, H. (2010). Trained lay first responders reduce trauma mortality: a controlled study of rural trauma in Iraq. Prehosp Disaster Med, 25(6), 533-539.

O'Muircheartaigh, C., A. Krosnick, J., \& Helic, A. (2000). Middle Alternatives, Acquiescence, and the Quality of Questionnaire Data. University of Chicago: Harris School of Public Policy Studies.

R Core Team. (2016). R: A language and environment for statistical computing. Vienna, Austria: R Foundation for Statistical Computing. Retrieved from https://www.R-project.org/

Rutterford, C., Copas, A., \& Eldridge, S. (2015). Methods for sample size determination in cluster randomized trials. Int J Epidemiol, 44(3), 1051-1067. doi:10.1093/ije/dyv113

Simpson, E. J. (1966). The classification of educational objectives: Psychomotor domain. IL J Home Econ., 10(4), 110-144.

Tannvik, T. D., Bakke, H. K., \& Wisborg, T. (2012). A systematic literature review on first aid provided by laypeople to trauma victims. Acta Anaesthesiol Scand, 56(10), 1222-1227. doi:10.1111/j.13996576.2012.02739.x

Teerenstra, S., Moerbeek, M., van Achterberg, T., Pelzer, B. J., \& Borm, G. F. (2008). Sample size calculations for 3-level cluster randomized trials. Clin Trials, 5(5), 486-495. doi:10.1177/1740774508096476

Van de Velde, S., Heselmans, A., Roex, A., Vandekerckhove, P., Ramaekers, D., \& Aertgeerts, B. (2009). Effectiveness of nonresuscitative first aid training in laypersons: a systematic review. Ann Emerg Med, 54(3), 447-457, 457 e441-445. doi:10.1016/j.annemergmed.2008.11.005

Wei, Y. L., Chen, L. L., Li, T. C., Ma, W. F., Peng, N. H., \& Huang, L. C. (2013). Self-efficacy of first aid for home accidents among parents with 0 - to 4-year-old children at a metropolitan community health center in Taiwan. Accid Anal Prev, 52, 182-187. doi:10.1016/j.aap.2012.12.002

WHO. (2016). Global Health Estimates 2015: Deaths by Cause, Age, Sex, by Country and by Region, 20002015. Retrieved from http://www.who.int/healthinfo/global burden disease/estimates/en/index1.html

Wisborg, T., Brattebo, G., Brinchmann-Hansen, A., \& Hansen, K. S. (2009). Mannequin or standardized patient: participants' assessment of two training modalities in trauma team simulation. Scand J Trauma Resusc Emerg Med, 17, 59. doi:10.1186/1757-7241-17-59

Zideman, D. A., Singletary, E. M., De Buck, E. D., Chang, W. T., Jensen, J. L., Swain, J. M., . . Yang, H. J. (2015). Part 9: First aid: 2015 International Consensus on First Aid Science with Treatment Recommendations. Resuscitation, 95, e225-261. doi:10.1016/j.resuscitation.2015.07.047

Zimmerman, B. J. (2000). Self-Efficacy: An Essential Motive to Learn. Contemp Educ Psychol, 25(1), 82-91. doi:10.1006/ceps.1999.1016 